\title{
Outcomes and associated ethical considerations of long-run pediatric ECMO at a single center institution
}

\author{
Guillermo J. Ares ${ }^{1,2} \cdot$ Christie Buonpane $^{1} \cdot$ Irene Helenowski ${ }^{3} \cdot$ Marleta Reynolds ${ }^{1} \cdot$ Catherine J. Hunter $^{1,3}$ (I)
}

Accepted: 21 January 2019 / Published online: 25 January 2019

c) Springer-Verlag GmbH Germany, part of Springer Nature 2019

\begin{abstract}
Purpose Survival of neonatal and pediatric patients undergoing extracorporeal membrane oxygenation (ECMO) $\geq 21$ days has not been well described. We hypothesized that patients would have poor survival and increased long-term complications. Methods Retrospective, single center, review and case analysis. Tertiary-care university children's hospital including neonatal, pediatric and cardiac intensive care units. After institutional review board approval, the charts of all patients $<18$ years of age undergoing ECMO for $\geq 21$ continuous days were performed, and they were compared to comparative patients undergoing shorter runs. Overall survival, incidence of complications, and post-discharge recovery were recorded.

Results Overall survival was $36 \%$ in patients undergoing $\geq 21$ days of ECMO $(N=14) .5 / 8$ patients with cardiopulmonary failure from acquired etiologies survived versus $0 / 6$ patients with congenital anomalies. $1 / 5$ survivors achieved complete recovery with no neurologic deficits. The remaining survivors suffer from multiple medical and neurodevelopmental morbidities.

Conclusion ECMO support for $\geq 21$ days is associated with poor survival, particularly in neonates with congenital anomalies. Long-term outcomes for survivors ought to be carefully weighed and discussed with parents given the high incidence of neurologic morbidities in this population.
\end{abstract}

Keywords Neonatal ECMO $\cdot$ Pediatric ECMO $\cdot$ Long-run $\cdot$ Neurodevelopmental outcome $\cdot$ Moral distress, Ethics

\section{Introduction}

Extracorporeal membrane oxygenation (ECMO) is a form of cardiopulmonary bypass used as a rescue intervention in patients with a high mortality index. It can lead to increased survival in neonates and children with certain, reversible respiratory and cardiac conditions; however, it is not without risks [1,2]. Adverse events include poor neurodevelopmental outcomes and chronic lung disease [3, 4]. Durations of greater than 21 days of ECMO therapy are generally considered to be "long-run". The use of "long-run" ECMO for

Catherine J. Hunter

Chunter@luriechildrens.org

1 Division of Pediatric Surgery, Ann and Robert H. Lurie Children's Hospital of Chicago, 211 E Chicago Avenue, Box 63, Chicago, IL 60611, USA

2 Department of Surgery, University of Illinois at Chicago, 840 South Wood Street, Suite 376-CSN, Chicago, IL 60612, USA

3 Feinberg School of Medicine, Northwestern University, 310 East Superior Street, Morton 4-685, Chicago, IL 60611, USA
$>21$ days is controversial in some centers and is thought to be associated with increased morbidity and decreased survival [5-7]. However, studies analyzing results and outcomes of patients in this subset are lacking. Moreover, in the case of prolonged ECMO there are ethical dilemmas regarding when to continue offering extracorporeal life support, and when to withdraw it [8-11]. The broad range of ethical issues associated with this include the concepts of uncertainty in clinical judgment, informed consent, medical futility and moral distress. Herein, we review all cases of long-run neonatal and pediatric ECMO from the past 10-years in a single center free-standing children's hospital. Our goals are to: (1) describe the survival rates of long-run ECMO, (2) evaluate long term morbidities, and (3) discuss the associated ethical issues surrounding the use of prolonged ECMO therapy. 


\section{Materials and methods}

\section{Subject search}

Following institutional review board approval institutional data for patients from birth to 18 years of age requiring ECMO at Ann \& Robert H. Lurie Children's Hospital from 2007 to 2017 were reviewed. Subjects of the study were selected using CPT Codes and ICD-9 codes (ICD-9 39.65, CPT 36822, 33960, 33961). Patients undergoing $\geq 21$ days of ECMO were identified as having more than 20 institutional procedure billing code 9131000 for daily ECMO services. We identified patients undergoing shorter runs ( $<14$ days and 14-20 days of ECMO) using similar strategy. Exclusion criteria included patients with diagnosis codes only but incomplete electronic medical records, and patients undergoing non-continuous days of ECMO were counted as separate runs, not additive durations.

\section{Data collection}

For each patient, we analyzed demographics, pre-existing comorbidities, medical insurance, estimated gestational age, age at the initiation of ECMO, ECMO duration, type of ECMO cannulation, procedural year, indication for ECMO support, and rationale for prolonging and discontinuing ECMO. Primary outcome was survival to hospital discharge as a surrogate marker of overall mortality. Survival to current date and post-ECMO follow-up duration were recorded. Secondary outcome measures included length of stay (LOS), medical comorbidities, ventilator dependence, and neurologic outcomes including intracranial hemorrhage, ischemic strokes, learning disability, developmental delays, sensory and motor dysfunction, and epilepsy. Patient electronic medical records were reviewed individually by two observers, with discussion and agreement on relevant data by a third investigator.

\section{Statistical analysis}

Included subjects were grouped as survivors versus non-survivors for data analysis. Categorical variables were compared using Fisher's exact test for statistical significance at the level of $p<0.05$ (SAS v9.4). Secondary outcomes are reported as observation without statistical analysis given low number of overall survivors.

\section{Results}

\section{Demographics}

Fourteen patients met study criteria and were included in the analysis, nine (64\%) were male. Five were white (36\%), seven black (50\%), and two (14\%) were of Hispanic ethnicity. The primary payer was commercial insurance in seven and government in the other seven. The mean age at the time of ECMO initiation was $1.5 \pm 2.2$ years, with half of the patients being less than 90 days old. The mean estimated gestational age was $39.2 \pm 1.0$ weeks with only one premature neonate, born at 24 weeks.

\section{Overall survival}

The overall survival for patients undergoing prolonged ECMO runs for $\geq 21$ days was $36 \%$. The clinical case details of the five survivors can be found in Table 1. Survival was $72 \%(N=141)$ for patients undergoing $<14$ days of ECMO and 50\% for 14-20 days $(N=20)$, as shown in Fig. 1. Patients initiated on ECMO at an older age ( $>90$ days) had higher rates of survival (Fig. 2). Only 1 of 7 (14\%) patients younger than 90 days old survived to hospital discharge versus 4 of 7 (57\%) patients older than 90 days old. Furthermore, all patients that survived to hospital discharge are still alive today, with a mean survival time of $4.4 \pm 2.9$ years.

Differences in survival for patients on ECMO $\geq 21$ days were analyzed based on various indications for ECMO (Fig. 3). A total of 5/8 patients (62\%) with cardiopulmonary failure from acquired etiologies (e.g., pneumonia, fulminant myocarditis) survived compared to $0 / 6$ survivors from patients with congenital conditions (e.g., hypoplastic left heart syndrome, congenital diaphragmatic hernia). A higher proportion of patients with acquired cardiac failure survived (2/2) compared to those with acquired etiologies for pulmonary failure (3/6). In our reference group undergoing 14-20 days of ECMO, 5/6 survivors had acquired etiologies of cardiopulmonary failure versus 5/14 with congenital conditions. When looking at all ECMO runs $\geq 14$ days, there was worse survival in patients with congenital pulmonary conditions $(2 / 12)$ versus acquired ones (7/10). Additionally, the rates of survival for patients on $\mathrm{ECMO} \geq 21$ days for organ recovery versus organ replacement were similar (36\% versus $33 \%)$. For patients with 14-20 days of ECMO, 15/20 patients were initiated for organ recovery versus $5 / 20$ for organ replacement. The organ recovery patients had lower survival rates (40\%) than the organ replacement patients (80\%). Figure 4 shows a Kaplan-Meier survival curve for the patients undergoing ECMO for organ recovery versus organ replacement.

The indication for discontinuing ECMO support was predictive of survival. All of the patients decannulated after a successful and progressive wean from ECMO support survived. On the contrary, five patients decannulated after attempted wean because of prolonged duration and two patients with circuit complications (i.e., severe bleeding or circuit thrombosis) did not survive.

There was no difference in survival between patients on veno-veno (VV) ECMO versus veno-arterial (VA) support, 
Table 1 Long-run ECMO survivor patient details

\begin{tabular}{|c|c|}
\hline \multicolumn{2}{|l|}{ Patient 1} \\
\hline Age/Gender & 7 year old female \\
\hline Indication & Acute fulminant myositis \\
\hline Cannulation & VA ECMO via femoral artery/vein \\
\hline Complications & Lower extremity ischemia, above the knee amputation \\
\hline Decannulation & Day 24 \\
\hline LOS & 88 days \\
\hline Follow-up & 3.5 years \\
\hline Current & Wheelchair bound, residual diastolic dysfunction \\
\hline \multicolumn{2}{|l|}{ Patient 2} \\
\hline Age/Gender & 5 year old male \\
\hline Indication & Fulminant myocarditis \\
\hline \multirow[t]{2}{*}{ Cannulation } & VA ECMO via internal jugular vein and carotid artery \\
\hline & $\begin{array}{l}\text { *LVAD placed ECMO day } 10 \text {. Poor pulmonary status necessitated } \\
\text { continued need for ECMO until transplant }\end{array}$ \\
\hline Complications & Ischemic, hemispheric stroke with hemorrhage \\
\hline Decannulation & Day 26 (received orthotropic heart transplant) \\
\hline LOS & 97 days \\
\hline Follow-up & 4.3 years \\
\hline Current & Hemiparesis, dialysis, $\mathrm{LD}, \mathrm{BD}$, suicidal ideation \\
\hline \multicolumn{2}{|l|}{ Patient 3} \\
\hline Age/Gender & 50-days-old male \\
\hline Indication & Pertussis, severe acute respiratory distress syndrome \\
\hline Cannulation & VA ECMO via internal jugular and carotid artery \\
\hline Complications & Seizures, hypoxic brain injury with multifocal hemorrhages \\
\hline Decannulation & Day 28 \\
\hline LOS & 66 days \\
\hline Follow-up & 8 years \\
\hline Current & Epilepsy, spastic hemiparesis, GD, min language comprehension \\
\hline \multicolumn{2}{|l|}{ Patient 4} \\
\hline Age/Gender & 13 month old male \\
\hline Indication & Pulmonary hemorrhage, pulmonary capillaritis \\
\hline Cannulation & VV ECMO via internal jugular vein \\
\hline Conversion & To VA ECMO due to declining cardiac status \\
\hline Complications & L temporal ICH, bronchoscopies with lavage and clot removal \\
\hline Decannulation & Day 32 \\
\hline LOS & 172 days \\
\hline Follow-up & 6 years \\
\hline Current & Vent-dependent, sensorineural hearing loss, cortical blindness, GD \\
\hline \multicolumn{2}{|l|}{ Patient 5} \\
\hline Age/Gender & 2 year old female \\
\hline Indication & Influenza $\mathrm{B}$, necrotizing pneumonia \\
\hline Cannulation & VV ECMO via *** \\
\hline Conversion & To VA ECMO for poor flows \\
\hline Complications & Tube thoracostomies for tension pneumothorax \\
\hline Decannulation & Day 25 \\
\hline LOS & 49 days \\
\hline Follow-up & 3 months \\
\hline Current & No neurologic deficits \\
\hline
\end{tabular}

$L D$ learning disability, $B D$ behavior disorder, $G D$ global developmental delay, $L$ left, $I C H$ intracranial hemorrhage, min minimal, vent ventilator 


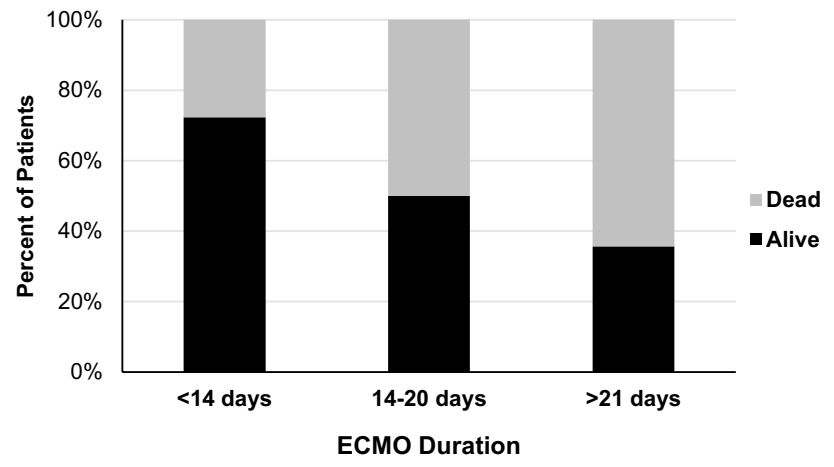

Fig. 1 Survival by ECMO Duration. Survival for patients grouped by duration of ECMO course. Survival was highest (72\%) in patients undergoing short runs $<14$ days $(102 / 141), 50 \%$ in patients with on ECMO for 14-20 days (10/20), and lowest (36\%) for prolonged ECMO runs $\geq 21$ days $(5 / 14)$ or between patients who were transitioned from VV to VA ECMO. Estimated gestational age, reason given for continuing ECMO > 21 days, LOS, or studied demographics were not significantly different between groups.

\section{Survivor morbidities}

Medical and neurologic outcomes of the 5 survivors are detailed in Table 2. The longest survival duration to date is 8 years. Three of five patients had intracranial hemorrhages (ICH) while on the ECMO circuit and 2 of 5 had ischemic strokes with residual hemiparesis. Moreover, 4 of the 5 patients have impaired ambulation secondary to severe contractures requiring serial interventions/bracing or amputation. A majority of patients (3/5) experienced significant developmental delay or learning disabilities. Only one of the five survivors, who was older at the time of ECMO and had an acquired pulmonary indication, had an intact survival from the standpoint of medical and neurologic disabilities.

Survivor morbidities were less severe in patients undergoing shorter ECMO runs of 14-20 days. Of the 10
Fig. 2 Survival by Age at Initiation of ECMO. Kaplan-Meier survival curve for patients on ECMO for $\geq 14$ days. For patients on $\mathrm{ECMO} \geq 21$ days, those older than 90 days of had higher rates of survival than those less than 90 days of age
Fig. 3 Survival by ECMO Indication. Survival for patients on ECMO runs $\geq 21$ days based on indication and etiology of cardiopulmonary failure. Patients with congenital pulmonary or heart conditions had overall poor survival compared to those with acquired conditions. Patients on ECMO for organ recovery $(n=11)$ and organ replacement $(n=3)$ had similar rates of survival
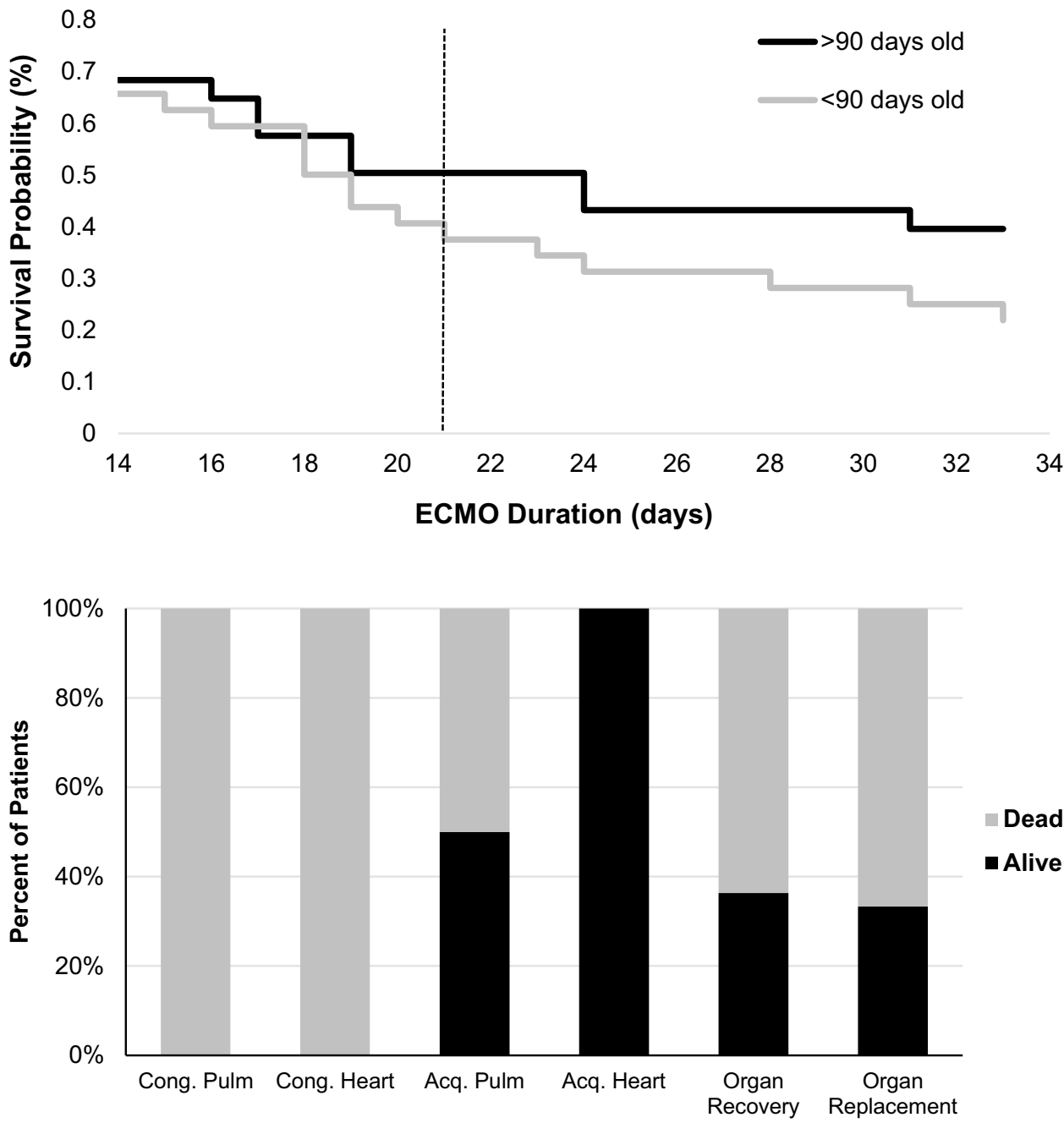
Fig. 4 Survival by ECMO Indication. Kaplan-Meier survival curve for patients on ECMO for $\geq 14$ days. For durations $>18$ days, patients on ECMO as a bridge to transplant had better survival than those on ECMO for organ recovery

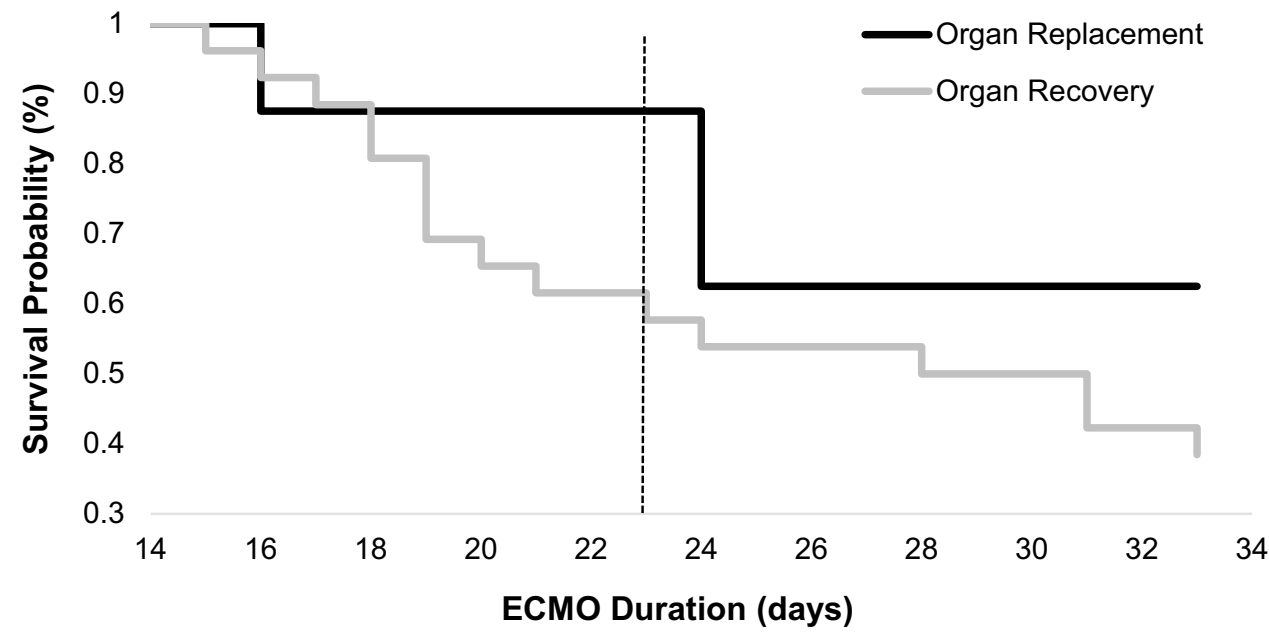

Table 2 Learning disability

\begin{tabular}{|c|c|c|c|c|c|}
\hline Patient & 1 & 2 & 3 & 4 & 5 \\
\hline Age at ECMO initiation & 7 years & 5 years & 50 days & 1 year & 2 years \\
\hline Current age (years) & 10 & 9 & 8 & 7 & 3 \\
\hline Indication & Fulminant myositis & Fulminant myositis & Pertussis pneumonia & Pulmonary hemorrhage & $\begin{array}{l}\text { Necrotizing } \\
\text { pneumo- } \\
\text { nia }\end{array}$ \\
\hline ECMO duration (days) & 24 & 26 & 29 & 32 & 25 \\
\hline $\mathrm{ICH}$ & - & $\mathrm{R}$ frontal $\mathrm{ICH}$ & Focal hemorrhage & L temporal hemorrhage & - \\
\hline Cardiovascular/Stroke & $\begin{array}{l}\text { Diastolic dysfunction } \\
\text { and CKD }\end{array}$ & $\begin{array}{l}\text { R MCA CVA with resid- } \\
\text { ual L-sided weakness }\end{array}$ & $\begin{array}{l}\text { Hypoxic brain injury } \\
\text { with residual R hemi- } \\
\text { paresis }\end{array}$ & - & - \\
\hline $\begin{array}{l}\text { Learning/ behavioral d } \\
\text { isability }\end{array}$ & - & $\begin{array}{l}\text { ADHD, delay in school, } \\
\text { suicidal ideation }\end{array}$ & $\begin{array}{l}\text { Global delay, begin- } \\
\text { ning to comprehend } \\
\text { language }\end{array}$ & $\begin{array}{l}\text { Global developmental } \\
\text { delay }\end{array}$ & - \\
\hline Sensory impairment & - & - & - & $\begin{array}{l}\text { Bilateral sensorineural } \\
\text { hearing loss and corti- } \\
\text { cal blindness }\end{array}$ & - \\
\hline Epilepsy & - & - & $\begin{array}{l}\text { Seizures since ECMO, } \\
\text { not well-controlled }\end{array}$ & - & - \\
\hline $\begin{array}{l}\text { Musculoskeletal mor- } \\
\text { bidities }\end{array}$ & $\begin{array}{l}\text { R BKA secondary to } \\
\text { ischemia from cannula }\end{array}$ & $\begin{array}{l}\text { Serial castings for pain- } \\
\text { ful contractures, poor } \\
\text { gait }\end{array}$ & $\begin{array}{l}\text { Serial castings and RLE } \\
\text { permanent bracing }\end{array}$ & $\begin{array}{l}\text { BLE weakness, ambula- } \\
\text { tory with assistance }\end{array}$ & - \\
\hline $\begin{array}{l}\text { Pulmonary/GI depend- } \\
\text { ence }\end{array}$ & - & - & - & Ventilator dependent & - \\
\hline
\end{tabular}

$L$ left, $R$ right, $I C H$ intracranial hemorrhage, $C K D$ chronic kidney disease, $M C A$ middle cerebral artery, $C V A$ cerebrovascular accident, $A D H D$ attention-deficit/hyperactivity disorder, $B K A$ below knee amputation, $B L E$ bilateral lower extremities, - none, N/A not available

survivors, five were intact from medical or neurologic disabilities. Three of the other five have an isolated medical problem: epilepsy (well-controlled), an upper extremity radial amputation for ischemia after cannulation, and feeding tube dependence for food aversion. One survivor has seizures, learning delay, and mild contractures but ambulates independently. Finally, one patient, who was at baseline dependent on ventilator support and tube feeds with hypotonia of prematurity, now has global developmental delay, hemiparesis, and is non-verbal post-ECMO.

Of the 14 patients on ECMO for $\geq 21$ days, nine did not survive. Three of the non-survivors were patients with congenital diaphragmatic hernias ( $\mathrm{CDH})$. One of them developed disseminated intravascular coagulation (DIC) with ICH and had to be decannulated on day of life 32 . One had a circuit thrombosis on ECMO day 21 and was decannulated. He 
also had evidence of ICH and seizures on ECMO. The third patient had CDH repair while on ECMO as an attempt to wean him off the circuit on day 23 , but succumbed to pulmonary hypertension. There were two patients with hypoplastic left heart syndrome, ages 6 months and 3 years, who were on ECMO awaiting donor heart for transplant. One of them had systemic hemorrhagic complications and circuit thrombosis on ECMO day 24, and the other one decompensated acutely on ECMO day 24 and developed multi-organ failure. Other patients had persistent pulmonary hypertension of the newborn, bronchopulmonary dysplasia complicated by bacterial pneumonia (age 14 months), and adenovirus pneumonia in a newborn, and respiratory syncytial virus (RSV) with bacterial superinfection. Ultimately, care was withdrawn for futility of medical treatment.

\section{Discussion}

ECMO is a life-sustaining and life-saving therapy for neonates and children with reversible cardiopulmonary failure. Although the majority of patients requiring ECMO are neonates and children and have ECMO runs of less than $200 \mathrm{~h}$ [12], a subset of patients are on ECMO for longer periods of time. Fortunately, the majority of patients undergoing ECMO at our institution do not require a prolonged course. However, in those that do, the outcomes and survival appear to be significantly worse after 21 days of ECMO therapy. Even with advancements in circuit management, both the overall outcomes and ECMO-related complications remain unchanged. Therefore, patient outcomes do not directly correlate with improvements in circuit management. Furthermore, patient outcomes are certainly affected by both baseline underlying disease and specific ECMO-related complications, although the weight of these factors is difficult to determine. In our study, we identified five long term survivors with a high association of post-ECMO major morbidity, resulting in a need for life-long ongoing medical and supportive care.

Although, there are recognized indications for an extended use of ECMO, such as a bridge to cardiac or pulmonary transplant $[13,14]$ other reasons to prolong ECMO duration (in the hope of clinical improvement) are less well defined. This uncertainty, coupled with a paucity of data regarding survivor outcomes contribute to ethical issues for both parents and health care providers. Although this study did not measure parental or provider attitudes, discrepancies in patient outcomes identify the need to consider ethical issues particular to long-run pediatric ECMO.

Initiation of ECMO is frequently considered emergent and initiated quickly. The clinical situation is typically of high acuity, and proceeding with ECMO is time sensitive. ECMO is often described as the "last hope" to sustain a critically ill child, who would otherwise almost certainly die without it. ECMO is considered when the mortality risk approaches $50 \%$ and indicated when the risk is in excess of $80 \%$ [15]. A health care provider's focus on the technical aspects of, "can we safely put this child onto ECMO" may outweigh the discussion of whether ECMO should be offered. Furthermore, an informed discussion regarding the end points of ECMO may be lacking. As surgeons, we may focus our discussion on the need to decannulate due to a major hemorrhagic event, or on the anticipation of clinical improvement meriting decannulation. However, the case of the child who fails to improve, but also maintains themselves adequately well for a prolonged period on the circuit may not be discussed in detail, or at all. Beauchamp listed five key elements of informed consent: Disclosure, comprehension, voluntariness, competence and consent [16]. Complete fulfillment of each of these elements is unlikely during an emotionally charged, time harried situation. There is rarely time for a prolonged conversation with the patient's family members, and not all invested parties may be present at the initial discussion. The exception is when a conversation regarding a potential need for ECMO is discussed prenatally, as for example, in the case of a fetus with a congenital diaphragmatic hernia and poor prognostic signs on prenatal imaging. Despite the recognized difficulties of adequate informed consent in emergent situations [17-19], health care providers should attempt to provide a succinct, understandable, and balanced review of the pertinent indications, benefits, alternatives, and associated risks and outcomes. Others have also suggested the early involvement of palliative care services to facilitate the needs of patients and their families during ECMO [20].

Removing a child from a circuit because of perceived futility is an especially difficult ethical dilemma for all involved. Defining futility is challenging, and universal agreement may be lacking among health care providers and clinical teams. The use of the term "futility" has been a topic of contentious debate among medical ethicists. In a statement from the Society of Critical Care Medical Ethics Committee, they define a medical intervention as, "considered inappropriate when there is no reasonable expectation that the patient will improve sufficiently to survive outside the acute care setting, or when there is no reasonable expectation that the patient's neurologic function will improve sufficiently to allow the patient to perceive the benefits of treatment" [21]. Whilst appearing to define clinical futility, the elements of clinical judgement and uncertainty remain. Not all persons may exhibit the same clinical judgement or have the same conclusions regarding the anticipated medical outcome. For example, the surgical team may determine that maintaining a child for greater than 21 days on venous arterial ECMO is futile, especially when prior clamp trials have failed and the patient exhibits minimal to no clinical 
improvement. Decannulation may be recommended, and death anticipated. Whereas the pediatric intensivist service may feel that there have been some clinical signs that portend improvement and warrant further ECMO support despite the failures described. When there is discord between clinical teams, or health care providers and parents moral distress may be experienced. Moral distress arises when difficult decisions are made in troubling situations [22]. The surgical group experiences moral distress because they believe that further support with ECMO is futile and that they are prolonging the child's suffering and extending false hope to the family regarding chances of survival. The intensivists experience moral distress because they feel that decannulation driven by concerns regarding ECMO duration, do not provide the child with a "fair chance" and that decannulation at this time may be "giving up" prematurely. By providing a more thorough analysis of the data of prolonged ECMO and its outcomes, we will be better able to predict likely endpoints. Although judgement in the face of clinical uncertainty will remain, the probability of making a correct assumption will be heightened. This may allow more harmonious clinical decision making and lessen moral distress for those involved.

Our study is limited by the number of patients identified in a single center review. However, the general trends identified particular patient groups at especially high risk of a poor outcome during a prolonged ECMO run. Future studies using larger database queries, such as the Extracorporeal Life Support Organization (ELSO) database, are warranted to support changes in current practice. Other authors have shown similar trends, though the detailing of long-term neurodevelopmental outcomes and medical morbidities of prolonged ECMO was unique of our study [23].

\section{Conclusions}

The high incidence of long term morbidity rates in those individuals who had an ECMO run time of greater than 21 days is concerning, and this information is valuable to provide counsel to parents of children with anticipated long-run ECMO. A larger ELSO database study would be beneficial to improve the power of this study and determine the impact of long-run ECMO on patient survival and outcomes nationally.

Acknowledgements This project was presented at The MacLean Center for Clinical Medical Ethics April 2018 and at the Academic Surgical Congress 2018.

Funding This study was funded by the National Institutes of Health NIDDK K08DK106450 $(\mathrm{CH})$ but did not receive any specific grant from funding agencies in the commercial, or not-for-profit sectors.

\section{Compliance with ethical standards}

Conflict of interest The authors declare that they have no conflict of interest.

Ethical approval All procedures performed in studies involving human participants were in accordance with the ethical standards of the institutional and/or national research committee and with the 1964 Helsinki declaration and its later amendments or comparable ethical standards. For this type of study formal consent is not required.

\section{References}

1. Andrews AF, Klein MD, Toomasian JM, Roloff DW et al (1983) Venovenous extracorporeal membrane oxygenation in neonates with respiratory failure. J Pediatr Surg 18(4):339-346

2. Glass P, Miller MShort B (1989) Morbidity for survivors of extracorporeal membrane oxygenation: neurodevelopmental outcome at 1 year of age. Pediatrics 83(1):72-78

3. Grover TR, Rintoul NEHedrick HL (2018) Extracorporeal membrane oxygenation in infants with congenital diaphragmatic hernia. Semin Perinatol 42(2):96-103

4. Prodhan P, Stroud M, El-Hassan N, Peeples S et al (2014) Prolonged extracorporeal membrane oxygenator support among neonates with acute respiratory failure: a review of the Extracorporeal Life Support Organization registry. ASAIO J 60(1):63-69

5. Green TP, Moler FWGoodman DM (1995) Probability of survival after prolonged extracorporeal membrane oxygenation in pediatric patients with acute respiratory failure. Extracorporeal Life Support Organization Crit Care Med 23(6):1132-1139

6. Brogan TV, Zabrocki L, Thiagarajan RR, Rycus PT et al (2012) Prolonged extracorporeal membrane oxygenation for children with respiratory failure. Pediatr Crit Care Med 13(4):e249-e254

7. Kays DW, Islam S, Richards DS, Larson SD et al (2014) Extracorporeal life support in patients with congenital diaphragmatic hernia: how long should we treat? J Am Coll Surg 218(4):808-817

8. Merrill ED, Schoeneberg L, Sandesara P, Molitor-Kirsch E et al (2014) Outcomes after prolonged extracorporeal membrane oxygenation support in children with cardiac disease-Extracorporeal Life Support Organization registry study. J Thorac Cardiovasc Surg 148(2):582-588

9. Partridge EA, Peranteau WH, Rintoul NE, Herkert LM et al (2015) Timing of repair of congenital diaphragmatic hernia in patients supported by extracorporeal membrane oxygenation (ECMO). J Pediatr Surg 50(2):260-262

10. Bein T, Weber-Carstens SHerridge M (2015) Extracorporeal life support, ethics, and questions at the bedside: how does the end of the pathway look? Intensive Care Med 41(9):1714-1715

11. Abdulhai S, Glenn IC, McNinch NL, Ponsky TA et al (2018) Current practices in the management of congenital diaphragmatic hernia patients requiring extracorporeal membrane oxygenation: results of an International Survey of Pediatric Surgeons. J Laparoendosc Adv Surg Tech A 28(5):606-609

12. Mahmood B, Newton DPallotto EK (2018) Current trends in neonatal ECMO. Semin Perinatol 42(2):80-88

13. Gulack BC, Hirji SAHartwig MG (2014) Bridge to lung transplantation and rescue post-transplant: the expanding role of extracorporeal membrane oxygenation. J Thorac Dis 6(8):1070-1079

14. Wallinder A, Pellegrino V, Fraser JFMcGiffin DC (2017) ECMO as a bridge to non-transplant cardiac surgery. J Card Surg 32(8):514-521 
15. Kukora SLaventhal N (2016) Choosing wisely: should past medical decisions impact the allocation of scarce ECMO resources? Acta Paediatr 105(8):876-878

16. Beauchamp TL (2011) Informed consent: its history, meaning, and present challenges. Camb Q Healthc Ethics 20(4):515-523

17. Brach $C$ (2016) Even in an emergency, doctors must make informed consent an informed choice. Health Aff (Millwood) 35(4):739-743

18. Boisaubin EVDresser R (1987) Informed consent in emergency care: illusion and reform. Ann Emerg Med 16(1):62-67

19. McCarthy DM, Leone KA, Salzman DH, Vozenilek JA et al (2012) Language use in the informed consent discussion for emergency procedures. Teach Learn Med 24(4):315-320

20. Doorenbos AZ, Starks H, Bourget E, McMullan DM et al (2013) Examining palliative care team involvement in automatic consultations for children on extracorporeal life support in the pediatric intensive care unit. J Palliat Med 16(5):492-495

21. Kon AA, Shepard EK, Sederstrom NO, Swoboda SM et al (2016) Defining futile and potentially inappropriate interventions: a policy statement from The Society of Critical Care Medicine Ethics Committee. Crit Care Med 44(9):1769-1774

22. Campbell SM, Ulrich CMGrady C (2016) A broader understanding of moral distress. Am J Bioeth 16(12):2-9

23. Cortina G, Niederwanger C, Klingkowski U, Velik-Salchner C et al (2018) Prolonged extracorporeal membrane oxygenation for pediatric necrotizing pneumonia due to Streptococcus pneumonia and influenza H1N1 co-infection: how long should we wait for native lung recovery? J Artif Organs 